\title{
Comparison of terbinafine and clotrimazole in treating tinea pedis
}

\author{
E G V Evans, B Dodman, D M Williamson, G J Brown, R G Bowen
}

Abstract

Objective-To compare the efficacy and safety of terbinafine $1 \%$ cream and clotrimazole $1 \%$ cream in the treatment of tinea pedis.

Design-Multicentre, double blind parallel group study.

Setting-32 general practices and one hospital.

Patients-256 patients with mycologically confirmed tinea pedis. Of the 211 patients evaluable, 107 were randomised to terbinafine ( 75 male, 32 female; mean (range) age 40 (12-81) years) and 104 to clotrimazole ( 79 male, 25 female; mean (range) age 36 (12-71) years).

Interventions-Terbinafine $1 \%$ cream applied twice daily for one week and inert cream applied twice daily for the next three weeks. Clotrimazole $1 \%$ cream applied twice daily for four weeks.

Main outcome measures-Mycological cure (negative results on microscopy and culture) and effective treatment (mycological cure plus no or minimal signs and symptoms) measured at weeks $1,2,3,4$, and 6.

Results-At week four rates of mycological cure were $93.5 \%$ for terbinafine and $73.1 \%$ for clotrimazole $(p=0 \cdot 0001)$; and at week six $97.2 \%$ for terbinafine and $83.7 \%$ for clotrimazole $(p=0.001)$. Rates of effective treatment at week 4 were $89.7 \%$ for terbinafine and $58.7 \%$ for clotrimazole $(p=0.0001)$; and $89.7 \%$ for terbinafine and $73 \cdot 1 \%$ for clotrimazole $(p=0.002)$ at week 6 .

Conclusion-These results indicate that a one

PHLS Mycology Keference Laboratory, Department of Microbiology, University of Leeds and General Infirmary, Leeds LS2 9JT E G V Evans, professor of medical mycology

Ackton Hospital, Ackton, Pontefract, West Yorkshire WF7 6HT

B Dodman, associate specialist in dermatology D M Williamson, consultant dermatologist

University Health Centre, Elms Road, Edgbaston, Birmingham B15 2SE G J Brown, general practitioner

Surgery, Penylan Road, Cardiff CF2 5HW

R G Bowen, general practitioner

Correspondence to:

Professor Evans.

$B M \mp 1993 ; 307: 645-7$ week course of terbinafine $1 \%$ cream is more effective in the treatment of tinea pedis than a four week course of clotrimazole $1 \%$ cream, both in terms of mycological cure and effective treatment.

\section{Introduction}

Tinea pedis (athlete's foot) is estimated to occur in $15 \%$ of the population with the highest incidence among regular users of communal bathing places. The most common and usually successful treatment for tinea pedis is a topical antifungal cream, which must be applied two to three times a day for up to four weeks; the prolonged treatment period probably reflects the primarily fungistatic action of these preparations. ${ }^{1}$ Although cure rates are good when these treatments are used as recommended, the number of applications required per day and the lengthy treatment period may lead to poor compliance by patients and failure of treatment. Clearly a topical treatment with a much shorter duration of treatment would be an advantage.

Terbinafine (Lamisil, Sandoz) is a synthetic antimycotic from the allylamine class of compounds that exerts a primary fungicidal action against dermatophytes and some other moulds and yeasts. ${ }^{23}$ Terbinafine $1 \%$ cream has been shown to be an effective treatment for various forms of dermatophyto- sis, candidiasis of the skin, and pityriasis versicolor. ${ }^{4}$ High rates of mycological cure (86-89\%) have been achieved in various forms of dermatophytosis with terbinafine $1 \%$ cream in placebo controlled studies by using once and twice daily applications for one to two weeks. ${ }^{5-9}$ In fact, recent studies suggest that durations of treatment of less than one week may be effective in tinea infections. ${ }^{1011}$

We present the results of a double blind study comparing twice daily application for one week of terbinafine $1 \%$ cream with twice daily application for four weeks of clotrimazole (Canesten, Baypharm), the most commonly prescribed topical antifungal in the United Kingdom for treatment of tinea pedis.

\section{Patients and methods}

PATIENTS AND TREATMENT

Males and females aged 12 or over with a clinical diagnosis of tinea pedis confirmed by positive results on microscopy were selected for inclusion in the study. Patients with a subsequent negative culture were classified as delayed exclusions. Written informed consent was obtained before entry from patients or their parents or guardians if they were below the age of consent. Women of childbearing age had to be using a reliable form of contraception. Patients were specifically excluded if their tinea pedis was of the "moccasin" or plantar type or if they suffered from concomitant onychomycosis, as these forms of dermatophytosis require treatment with oral antifungals. Any patients who had received systemic antimycotic treatment in the preceding six weeks or had received topical antifungal or potassium permanganate treatment within the past seven days were excluded. Patients were specifically asked about the use of over the counter antifungal preparations but were allowed to continue taking concomitant medication for indications other than fungal infections.

The number of patients recruited to the study was based on a calculation of sample size such that the study would have an adequate power to show with $95 \%$ confidence that there was a maximum difference of $15 \%$ between terbinafine and clotrimazole. Patients were then randomised (by using Fisher and Yates tables) to receive either twice daily treatment of terbinafine $1 \%$ cream for one week followed by three weeks of placebo cream or twice daily treatment of clotrimazole $1 \%$ cream for four weeks. The two antifungal and the placebo creams were packed in identical tubes to maintain the double blind nature of the study.

Patients were screened one week before entry and those with positive results on microscopy were entered into the study. Patients returned for assessments at weeks one, two, and three, at which they returned the previous week's medication and were dispensed the next week's medication; this ensured that the active terbinafine cream was not being used for longer than 
one week. Patients also returned for assessment at week four (end of treatment) and at week six (two weeks after the end of treatment).

MYCOLOGICAL AND CLINICAL ASSESSMENTS

At each week a skin scraping was taken and sent to a central laboratory in Leeds for mycological investigation. This consisted of direct microscopy in $20 \%$ potassium hydroxide and culture on Sabouraud glucose agar (plus chloramphenicol $0.05 \%$, actidione $0.5 \%$, w:v) at $27^{\circ} \mathrm{C}$ for up to two weeks. Any signs and symptoms of infection-namely, erythema, scaling, vesiculation, pustules, crusting, and pruritus-were rated by the physician on the scale $0=$ absent, $1=$ mild, 2 =moderate, 3 =severe, to give a clinical score (maximum score of 18). A rating was also made of a target lesion (identified at week 0 and being the largest and most severely affected area) by using the same scale. Any adverse events were recorded and scored on the scale of $1=$ mild, $2=$ moderate, $3=$ severe.

Patients were classified as mycologically cured if they had negative results on microscopy and culture. Mycologically cured patients with a total signs and symptoms score of $\leqslant 2$ (providing this was made up of two "milds" and not one "moderate") were classified as "effectively treated." All other outcomes were classified as "ineffectively treated." Analysis was carried out on an intention to treat basis. Efficacy and mycology results were assessed as follows. For each treatment group the percentage of patients cured was determined. The difference between groups and corresponding $95 \%$ confidence intervals was calculated. As the data was of a non-parametric nature, the MantelHaenszel $\chi^{2}$ test was used to test for significance between treatment groups.

\section{Results}

Two hundred and fifty six patients with tinea pedis were entered into the study. Of these, 41 were classified as delayed exclusions ( 38 patients with negative baseline mycology and three with non-dermatophyte fungal infections). One patient using clotrimazole for paronychia was excluded from the analysis as were three patients who failed to return after the entry visit.

Therefore 211 patients were evaluable, of whom 107 were randomised to terbinafine ( 75 male, 32 female; mean (range) age $40(12-81)$ years) and 104 to clotrimazole (79 male, 25 female; mean (range) age 36 (12-74) years). Table I shows the infecting organisms, and, as expected, the most common causal agent was Trichophyton rubrum.

The mycological cure rates are shown in table II and

TABLE I-Number of patients with tinea pedis according to infecting organism and allocation of treatment

\begin{tabular}{lcc}
\hline Infecting organism & Terbinafine & Clotrimazole \\
\hline Trichophyton rubrum & 68 & 79 \\
Trichophyton mentagrophytes & 31 & 22 \\
Epidermophyton flocossum & 8 & 2 \\
Dermatophyte unspecified & 0 & 1 \\
\hline Total & 107 & 104 \\
\hline
\end{tabular}

TABLE II-Numbers (percentages) of patients mycologically cured (negative microscopy and culture) at each visit

\begin{tabular}{lccc}
\hline Week & $\begin{array}{c}\text { Terbinafine } \\
(\mathrm{n}=107)\end{array}$ & $\begin{array}{c}\text { Clotrimazole } \\
(\mathrm{n}=104)\end{array}$ & $\begin{array}{c}\text { Percentage difference } \\
(95 \% \text { confidence } \\
\text { interval })\end{array}$ \\
\hline 1 & $42(39)$ & $40(39)$ & $0 \cdot 8(-12 \cdot 4$ to $14 \cdot 4)$ \\
2 & $75(70)$ & $56(54)$ & $16 \cdot 3(3.4$ to $29 \cdot 2)$ \\
3 & $90(84)$ & $74(71)$ & $12 \cdot 9(1 \cdot 9$ to $23 \cdot 9)$ \\
4 & $100(94)$ & $76(73)$ & $20 \cdot 4(10 \cdot 7 \text { to } 30 \cdot 1)^{\star}$ \\
6 & $104(97)$ & $87(84)$ & $13 \cdot 5(5 \cdot 8$ to $21 \cdot 2) \dagger$ \\
\hline${ }^{\star} \mathrm{p}=0 \cdot 0001$. & & & \\
$\mathrm{tp}=0.001$. & & &
\end{tabular}

TABLE III-Numbers (percentages) of patients effectively treated (mycologically cured and no or minimal signs and symptoms) at each visit

\begin{tabular}{lccc}
\hline Week & $\begin{array}{c}\text { Terbinafine } \\
(\mathrm{n}=107)\end{array}$ & $\begin{array}{c}\text { Clotrimazole } \\
(\mathrm{n}=104)\end{array}$ & $\begin{array}{c}\text { Percentage difference } \\
(95 \% \text { confidence } \\
\text { interval })\end{array}$ \\
\hline 1 & $20(19)$ & $9(9)$ & $10 \cdot 0(0 \cdot 8$ to $19 \cdot 4)$ \\
2 & $54(51)$ & $24(23)$ & $27 \cdot 4(14 \cdot 9$ to $39 \cdot 9)$ \\
3 & $83(78)$ & $50(48)$ & $29 \cdot 5(17 \cdot 1$ to $41 \cdot 9)$ \\
4 & $96(90)$ & $61(59)$ & $31 \cdot 0(19 \cdot 9 \text { to } 42 \cdot 1)^{\star}$ \\
6 & $96(90)$ & $76(73)$ & $16.6(6.5$ to $26 \cdot 9) \dagger$ \\
\hline${ }^{\star} \mathrm{p}=0.0001$. & & &
\end{tabular}
$\mathrm{tp}=0.002$.

in the figure. Mycological cure was achieved more rapidly on terbinafine compared with clotrimazole, with over $70 \%$ (75) of patients treated with terbinafine mycologically cured after only two weeks. At week 4 (end of treatment) mycological cure rates with terbinafine were $94 \%$ (100) compared with $73 \%$ (76) with clotrimazole, a significant difference $(p=0.0001)$. Similarly at week 6 (two weeks after the end of treatment) mycological cure rates with terbinafine were significantly better than with clotrimazole $(97 \%$ (104) $v 84 \%(87), \mathrm{p}=0.001)$.

The numbers and percentages of patients effectively treated are shown in table III and in the figure. The proportion of patients effectively treated by terbinafine was higher at each visit compared with clotrimazole. At four weeks $90 \%$ (96) of patients were effectively treated by terbinafine compared with only $59 \%$ (61) by clotrimazole $(p=0.0001)$. At week 6 the proportion of patients effectively treated by terbinafine remained unchanged but was still significantly higher than the $73 \%(76)$ of patients effectively treated by clotrimazole $(\mathrm{p}=0.002)$.

Four adverse events in the terbinafine group were probably or certainly related to treatment. These included painful stinging and cracks, increased itching, irritation of eyes, and erythema or swelling of skin; one patient stopped terbinafine treatment because of erythema or swelling of skin. In the clotrimazole group three adverse events were probably or certainly related to treatment. Signs and symptoms experienced included erythema, soreness, and a red rash. One patient with cellulitis of the foot, which was probably unrelated to treatment, stopped using clotrimazole.

\section{Discussion}

In this study twice daily applications of terbinafine $1 \%$ cream for one week (followed by three weeks of placebo) was significantly superior, both in terms of mycological cure and effective treatment, to twice daily applications for four weeks of clotrimazole when compared six weeks after starting treatment. The cure rates with terbinafine $1 \%$ cream were high, with $97 \%$ of patients mycologically cured and $90 \%$ of patients effectively treated. Two weeks after the end of treatment with clotrimazole, however, the cure rates were still rising and if the follow up period had been longer they may have been higher. It is also worth noting that five weeks after the completion of treatment with terbinafine the mycological cure rates were still rising, confirming the findings of other studies which suggest that short duration treatment with terbinafine is not associated with relapse. ${ }^{9}$ Nevertheless, one must acknowledge that had the follow up period been longer in this study relapses might have occurred, but then the difficulty would have been in distinguishing these relapses from cases of reinfection.

The superiority of terbinafine over clotrimazole, even with a shorter duration of treatment, is probably because terbinafine has a primarily fungicidal mode of action whereas clotrimazole is primarily fungistatic. One has to recognise, however, the theoretical possi- 


\section{Clinical implications}

- Tinea pedis (athlete's foot) occurs in about $15 \%$ of the population

- Its usual treatment is a topical antifungal applied two or three times a day for up to four weeks

- Terbinafine is a synthetic allylamine antifungal with a primarily fungicidal action against dermatophytes and some other moulds and yeasts

- This study shows that terbinafine $1 \%$ cream applied twice daily for a week was significantly more effective in treating tinea pedis than a four week course of clotrimazole $1 \%$ cream, both clinically and mycologically

- Because it has a high efficacy in the short term terbinafine may become the preferred topical treatment for tinea pedis

bility that the use of placebo cream for three weeks after active treatment in the terbinafine group (to make the study double blind) may have in some way enhanced the cure rate, although any such effect is likely to have been minimal.

A new topical antifungal agent needing fewer applications and a shorter duration of treatment would clearly be advantageous and likely to improve compliance by patients provided that efficacy is not compromised. Results to date have shown that reducing the duration of treatment with terbinafine $1 \%$ cream to one week in tinea infections does not compromise efficacy. ${ }^{5-9}$ This study further confirms these findings but more importantly shows that one week's terbinafine $1 \%$ cream is actually significantly more effective than the recommended four weeks' treatment with clotrimazole $1 \%$ cream in tinea pedis. The cost implications are that one week's treatment with terbinafine $1 \%$ cream costs $£ 4.98$ (15 g tube) compared with $£ 4.26$ for four weeks' treatment with clotrimazole $1 \%$ cream ( $50 \mathrm{~g}$ tube). Possibly terbinafine $1 \%$ cream will become the preferred topical treatment of tinea pedis.

We thank the following colleagues for their contribution to the study and for allowing us to use their data: R C Goodfellow, Preston; R D Rowley and S Gibeon, London; A Bevan, Potters Bar; R Cranfield and R J Baldwin, Gwent; R B Lamberton, Aberdeen; J P Heatley, Horsham; R Cantor, C A Maxwell, and D M Entwistle, Cardiff; R C Joshipura, S K Chouksey, J M Shah, and C S Joshipura, Manchester; A K Mishra, Lancashire; K Brown, Coppull; R Thrush, Walsall; J Adams, T J Mallon, and R N Stones, Cheshire; P M Baldwin, Nottingham; H MacKinnon and J R Ross, Glasgow; R A J Seaman, Hitchin; P C Heaney, Northampton; G J Brown, Birmingham; I G V James, Bolton; B K Jaiswal, Essex; T F Poyner, Cleveland. We thank Mr R A Forster for help with the mycological investigations and Sandoz Pharmaceuticals (UK) for supplying the terbinafine and supporting the study.

1 Sud IJ, Fenigold DS. Mechanisms of action of the antimycotic imidazoles. f Invest Dermatol 1981;76:438-41.

2 Petranyi G, Meingassner JG, Meith $\mathrm{H}$. Antifungal activity of the allylamine derivative terbinafine in vitro. Antimicrob Agents Chemother 1987;31:1365-8.

3 Clayton YM. In vitro activity of terbinafine. Clin Exp Dermatol 1989;14:101-4.

4 Villars V, Jones TC. Clinical efficacy and tolerability of terbinafine (Lamisil) a new topical and systemic fungicidal drug for treatment of dermatomycoses. Clin Exp Dermatol 1989;14:124-7.

5 Millikan LE. Efficacy and tolerability of topical terbinafine on the treatment of tinea cruris. 7 Am Acad Dermatol 1990;23:795-9.

6 Greer DL, Jolly HW Jr. Treatment of tinea cruris with topical terbinafine. IAm Acad Dermatol 1990;23:800-4.

7 Evans EGV, James IGV, Joshipura RC. One week treatment of tinea corporis and tinea cruris with terbinafine (Lamisil) $1 \%$ cream: a placebo controlled study. Fournal of Dermatological Treatment 1992;3:181-4.

8 Evans EGV, James IGV, Joshipura RC. Two week treatment of tinea pedis with terbinafine (Lamisil) 1\% cream: a placebo-controlled study. foumal of Dermatological Treatment 1991;2:95-7.

9 Berman B, Ellis C, Leydon J, Lowe N, Savin R, Shupack J, et al. Efficacy of a 1 week, twice daily regimen of terbinafine $1 \%$ cream in the treatment of interdigital tinea pedis. $\mathcal{f} \mathrm{Am}$ Acad Dermatol 1992;26:956-60.

10 Hill S, Thomas R, Smith SG, Finlay AY. An investigation of the pharmacokinetics of topical terbinafine (Lamisil) 1\% cream. Br $\mathcal{F}$ Dermatol 1992;127: $396-400$

11 Evans EGV. A double-blind comparison of 1, 3, 5 and 7 day topical therapy with $1 \%$ terbinafine (Lamisil) cream in tinea pedis. $\mathrm{Br} \mathcal{f}$ Dermatol 1992;127:21.

(Accepted 22 fune 1993)

\title{
Psychiatric consequences of road traffic accidents
}

\author{
Richard Mayou, Bridget Bryant, Robert Duthie
}

Abstract

Objective-To determine the psychiatric consequences of being a road traffic accident victim.

Design-Follow up study of road accident victims for up to one year.

Setting-Emergency department of the John Radcliffe Hospital, Oxford.

Subjects-188 consecutive road accident victims

University Department of Psychiatry, Warneford Hospital, Oxford OX3 7JX Richard Mayou, clinical reader in psychiatry Bridget Bryant, research officer

Nuffield Department of Orthopaedic Surgery, Nuffield Orthopaedic Centre, Oxford

Robert Duthie, professor

Correspondence to:

Dr Mayou.

BMF 1993;307:647-51 aged 18-70 with multiple injuries (motorcycle or car) or whiplash neck injury, who had not been unconscious for more than 15 minutes, and who lived in the catchment area.

Main outcome measures-Present state examination "caseness"; post-traumatic stress disorder and travel anxiety; effects on driving and on being a passenger.

Results-Acute, moderately severe emotional distress was common. Almost one fifth of subjects, however, suffered from an acute stress syndrome characterised by mood disturbance and horrific memories of the accident. Anxiety and depression usually improved over the 12 months, though one tenth of patients had mood disorders at one year. In addition, specific post-traumatic symptoms were common. Post-traumatic stress disorder occurred during follow up in one tenth of patients, and phobic travel anxiety as a driver or passenger was more common and frequently disabling. Emotional disorder was associated with having pre-accident psychological or social problems and, in patients with multiple injuries, continuing medical complications. Post-traumatic syndromes were not associated with a neurotic predisposition but were strongly associated with horrific memories of the accident. They did not occur in subjects who had been briefly unconscious and were amnesic for the accident. Mental state at three months was highly predictive of mental state at one year.

Conclusions-Psychiatric symptoms and disorder are frequent after major and less severe road accident injury. Post-traumatic symptoms are common and disabling. Early information and advice might reduce psychological distress and travel anxiety and contribute to road safety and assessing "nervous shock."

\section{Introduction}

Road traffic accidents are a major cause of morbidity and are the main cause of death in people under 30 . 